Studies in African Linguistics

Volume 33, Number 1, 2004

\title{
ESQUISSE DE LA TONOLOGIE SYNCHRONIQUE DU WEMEGBE, DIALECTE GBE DU SUD-BÉNIN*
}

\author{
Flavien Gbeto \\ Université d'Abomey-Calavi
}

In this paper I show that Wemegbe, a language spoken in southern Benin, has two underlying tones, $\mathrm{H}$ and $\mathrm{L}$, and that the surface tones $\mathrm{M}, \mathrm{LH}$ and $\mathrm{HL}$ are derived by phonological rules. A $\mathrm{H}$ tone is inserted after an initial prevocalic voiceless obstruent (occurring in non verbals), creating a HL contour tone, when the vowel of the syllable is $\mathrm{L}$ toned. The LH tone is derived through a rightspreading rule from a L' prefix tone, which is postulated for all verbs in their imperative forms and for all nouns. Of particular interest is the fact that the rightward spreading rule for $\mathrm{L}^{\prime}$ is blocked by a voiceless obstruent. Finally, the $\mathrm{M}$ tone is derived through a L-Raising rule. This analysis shows that not only can vowels be TBUs in the world's languages, but also initial root consonants can be.

\section{Introduction}

Le wemegbe est parlé dans la province de l'Ouémé, sur tout le territoire des districts ruraux de bonu et d'ajowun, dans le district de: dangbo (sauf dans les com-

* Je voudrais remercier l'éditeur de SAL, David Odden, ainsi que le lecteur anonyme pour leurs remarques et suggestions. Je remercie aussi Sayane Gouroubéra pour sa lecture assidue du texte. Ces remarques m'ont imposé un travail supplémentaire de terrain; travail qui a sans doute permis, je l'espère, d'améliorer la qualité de l'article tant du point de vue du fond que de la forme. Ils ne doivent pas pourtant être tenus responsables des lacunes du texte final. Je remercie également mes informateurs Hounsou Eric, Dansou Honoré, Houmênou André, tous du village de Agonlin-Lowé ainsi que feue Azonhoumon Bertille, originaire du village d'Akpadanou pour la patience dont ils ont fait preuve en me livrant des données précieuses sur leur langue. 
munes de deken et de gbeko), dans les communes d'avagboji (district rural des agege), de zungbome (district rural d'akpro-miserete) et de jigbe (district rural de ze), et dans le village de katagon (district d'avlanku) (voir CNL 1983: 61). Le parler du chef lieu de la commune d'Adjohoun a été déjà l'objet d'un travail de mémoire de maîtrise (voir Houndékan 1983). Dans cet article, le parler que j'étudie est celui des villages d'Agonlin-Lowé et d'Akpadanou, commune d'Adjohoun, département du Plateau. Il sera glosé sous le terme wemegbe. Le weme est partie intégrante de la soixantaine de dialectes gbe (voir Capo 2000) appartenant au Left Bank (Stewart 1989) du New Kwa selon la classification de Williamson (1989).

L'article sera organisé comme suit: dans la section 1, je présenterai le système consonantique de la langue, puisque les réalisations tonales dans cette langue dépendent de la nature de la consonne. Dans la section 2, je présenterai les structure syllabiques de la langue. Dans la section 3, je présenterai le point de la méthodologie. La section suivante sera consacrée aux tons dans le système nominal. Dans cette partie, on procèdera à une analyse distributionnelle des tons identifiés, analyse que je terminerai par le tableau récapitulatif de la distribution des tons dans le système nominal. La section 5 sera consacrée à la distribution des tons dans le système verbal. Quant à la section 6, elle sera consacrée à l'analyse. On y montrera surtout que le weme a deux tons inhérents $\mathrm{H}$ et non-H symbolisés par $\mathrm{H}$ et $\mathrm{B}$. Les tons $\mathrm{M}, \mathrm{HB}$ et $\mathrm{BH}$ sont dérivés de ces deux tons de base. Ainsi, il sera montré que le ton $\mathrm{M}$ est une réalisation du ton $\mathrm{B}$ (6.2.2.1). Dans la soussection 6.2.1, on montrera que le ton $\mathrm{BH}$ est dérivé par une règle de propagation préfixal B' présent dans la forme sous-jacente de tout radical (nominal et verbal). La propagation du ton préfixal $\mathrm{B}^{\prime}$ a lieu en présence des consonnes voisées. Elle est bloquée par les obstruantes non voisées. Quant au ton HB relevé uniquement au niveau des nominaux, on montrera qu'il est dérivé par une règle d'insertion du ton consonantique $\mathrm{H}$ devant ton $\mathrm{B}$ après obstruante non voisée. La section 7 fera le point des idées discutées.

J'adopterai, dans ce travail, le système de transcription de l'API (Alphabet Phonétique International), sauf pour la notation des fricatives uvulaires notées [x] et [h], pour la notation de la nasale et de la semi-consonne palatale notée respectivement [ny] et [y]. Les tons seront notés comme suit: le ton $\mathrm{B}$ (as) par un accent grave ('), le ton $\mathrm{H}$ (aut) par un accent aigu ('), le ton M(oyen) par une barre horizontale $\left({ }^{-}\right)$, le ton montant $\overparen{\mathrm{BH}}$ par le signe $\left({ }^{\vee}\right)$ et le ton descendant $\overparen{\mathrm{HB}}$ par le signe $\left(`^{\wedge}\right.$ ) sur la voyelle. Contrairement à la pratique de l'API, la nasalité sera transcrite, pour des raisons d'esthétique, par un tilde souscrit à la voyelle. Sauf précision, toute transcription doit être considérée comme phonétique 


\section{Le Système Consonantique du Wemєgbe.}

Comme la plupart des langues gbe, le wemegbe distingue douze voyelles phonologiques: /i, e, $\varepsilon, \mathrm{a}, \mathrm{\jmath}, \mathrm{o}, \mathrm{u}, \mathrm{i}, \underset{\sim}{\varepsilon}, \underset{\sim}{\mathrm{a}}, \underset{\sim}{\mathrm{j}}, \mathrm{\sim} / \mathrm{.}$. Le système consonantique présente les unités suivantes résumées au tableau 1 :

\section{Tableau 1: Phonèmes consonantiques du weme}

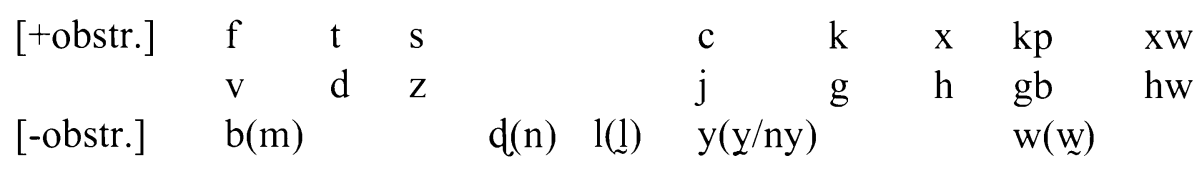

Le tableau 1 présente une opposition [+obstruant]/[-obstruant].

Au niveau des obstruantes, il y a une opposition de voisement. Synchroniquement, tous les dialectes gbe opposent les obstruantes non voisées aux obstruantes voisées (Capo 1991). Au niveau des [-obstruant] qui sont toutes redondamment voisées, on note un phénomène de distribution complémentaire entre consonnes orales et consonnes nasales; les consonnes nasales [m, n, $, \mathrm{y} / \mathrm{ny}, \mathrm{w}]$ apparaissant avant voyelles nasales et leurs orales correspondantes [b, d, l, y, w] uniquement avant voyelles orales. Suivant la tradition inauguree par Capo (1981, 1983, 1991), Bole-Richard (1983) et Gbéto (1997a), je considère les consonnes nasales [m, n, 1, ny, w] comme étant des variantes combinatoires prévisibles de $/ \mathrm{b}$, $\mathrm{d}, 1, \mathrm{y}, \mathrm{w} /$ devant voyelles nasales.

Lorsqu' on observe le comportement des consonnes [-obstruant] et des [+obstruant] par rapport aux faits de tonalité (voir Gbéto 2002b), on se rend compte qu'elles se répartissent en un certain nombre de classes naturelles de sons. Cette observation a été faite dans la littérature sur le gbe depuis Ansre (1961). On distingue notamment en gbe: les obstruantes voisées notées $T$ et les obstruantes non voisées notées $\mathrm{D}$. Les consonnes de la classe $\mathrm{D}$ ont pour effet de rabaisser le ton suivant alors que celles de la classe $\mathrm{T}$ ont pour effet de le rehausser. Le comportement des consonnes [-obstruant] est problématique en gbe (voir Gbéto $2002 b, 2003)$. Ces consonnes se répartissent selon les trois classes naturelles suivantes (voir Gbéto 2002b): la classe des approximantes comprenant $/ \mathrm{l}, \mathrm{y}, \mathrm{w} /$ et notée $\mathrm{L}$; la classe des sonantes nasales comprenant [m, n, ny, y, l, w] et notée $\mathrm{N}$, et la classe des consonnes implosives comprenant $/ \mathrm{b}, \mathrm{d} /$ et notée $\mathrm{B}$.

Si l'on en croit Stewart (1989, 1994), cette disparité est le résultat des changements phonétiques qui ont permis de dériver les proto-segments du gbe de ceux du proto-Volta-Congo, ancêtre des langues gbe. En proto-Volta-Congo, les 
consonnes sonores douces, i.e. ['b, 'd, 'j, 'gb, l] sont en distribution complémentaire avec les nasales correspondantes [m, n, n, nm, 1]; les douces et les sonantes apparaissant respectivement devant voyelles orales et nasales. Stewart (1994: 185-187) propose alors une première règle qui remplace les dorsales douces voisées (i.e. les dorsales implosives et leurs partenaires nasales) par des semi-voyelles $[\mathrm{y}, \mathrm{w}, \mathrm{y}, \underset{\sim}{\mathrm{w}}]$. A cette étape de la dérivation, toutes les consonnes concernées, à l'exception de ['b, 'd], sont des sonantes. Une deuxième règle remplace les deux consonnes restantes par $[\mathrm{b}, \mathrm{d}]$. Deux conclusions peuvent être tirées de ce schéma. Le premièr est que les consonnes implosives ['b, 'd] du proto-Volta-Congo n'ont pas survécu en proto-gbe. Elles ont été remplacées par [b, d], i.e. des consonnes obstruantes voisées. Gbéto (2002b) a montré que ces consonnes peuvent avoir un effet sur la réalisation tonale suivante en abaissant, comme les obstruantes voisées, la réalisation tonale suivante. Le deuxièm est que les consonnes implosives ['b, 'd] n'ont pas disparu sans laisser de trace en protogbe. En effet, elles continuent de constituer, avec les sonantes, une classe naturelle de sons qui n'en est pas une. C'est donc cette évolution aberrante qui explique le comportement bizarre et des sonantes et des consonnes [b, d].

Toute étude tonale sérieuse sur le gbe doit prendre en compte les évolutions constatées, puisqu'elles ont des répercussions sur les réalisations tonales synchroniques. En wemegbe par exemple, si l'on tient compte du phénomène tonal, deux classes naturelles de sons sont à prendre en considération: la classe des [+obstruant, -voix] et celle des [C, +voix] comportant aussi bien les obstruantes voisées que les sonantes, ainsi que les consonnes [b, d].

Gbéto (2002b) a montré que cette répartition tient compte de la propriété qu'ont ces consonnes de favoriser ou de ne pas favoriser l'application des règles phonologiques. Ainsi, devant les sonantes (orales ou nasales), le ton $\mathrm{H}$ peut s'abaisser; alors que le ton $\mathrm{B}$ peut se rehausser. Ce relèvement et cet abaissement peuvent dépendre aussi du caractère oral ou nasal de la sonante en question. Devant $[b, d]$, le ton $H$ peut s'abaisser; il arrive que ces consonnes n'aient aucune influence sur la réalisation tonale suivante, reflet de l'histoire de la langue.

\section{Structures Syllabiques}

2.1 Structure canonique. La structure syllabique minimale d'un mot (nominal et verbal) est le suivant (voir Gbéto 1997a, b) en gbe. Westermann $(1927,1930)$ a insisté sur la forte tendance des langues gbe au monosyllabisme. 
(1)

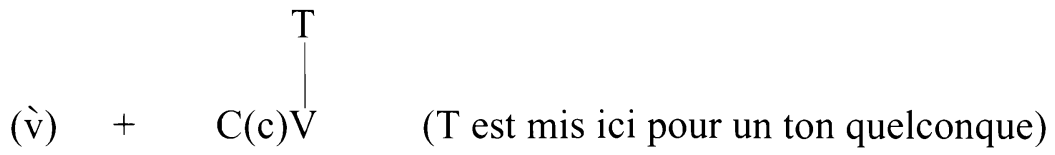

La formule (1) comporte une voyelle initiale et une base radicale de forme $\mathrm{C}(\mathrm{c}) \mathrm{V}$.

La voyelle initiale, qui peut être toutes les voyelles orales en gbe sauf $[u],{ }^{1}$ sera considérée comme le préfixe vocalique. Ce préfixe vocalique porte toujours un ton non Haut, c'est-à-dire B en wemegbe. Westermann $(1927,1930,1947)$ considère les voyelles initiales en gbe comme un vestige de classes nominales. Mukarovsky (1969) a tenté de rapprocher les préfixes vocaliques de l'ewe (entendez gbe) des préfixes nominaux des langues à classes comme le gurma, les langues bantu, le guang, le twi, le moore. Bien qu'il n'y ait pas accord de classes comme dans les langues à classes (comme les langues gur ou bantu), j'adopte la terminologie consacrée en gbe qui est de considérer les voyelles initiales comme étant des préfixes nominaux. Les préfixes nominaux distinguent formellement un nominal d'un verbal. Le verbe commence donc toujours, en surface, par une consonne. D'un point de vue phonologique pourtant, je considère, à la suite de Stahlke (1971a, b), Capo (1977) et Gbéto (1995, 1997a, 2002b) que le verbe est composé de deux éléments: un ton flottant $\mathrm{B}^{\prime}$ (qui est le morphème de l'impératif) et un radical indiquant la forme neutre (infinitive) du verbe:

(2) Nominal

làdú/ [àdǔ] 'dent' lòtó/ [òtó] 'oreille'

(3) Verbal

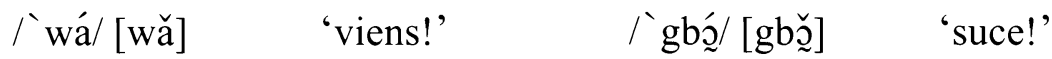

Le radical $\mathrm{C}(\mathrm{c}) \mathrm{V},{ }^{2}$ où $c$ peut être n'importe laquelle des approximantes $/$, $\mathrm{y}, \mathrm{w} /$. Les conditions de distribution de $c$ par rapport à $C$ (qui théoriquement peut être n'importe quelle consonne du système phonologique) n'étant pas pertinentes pour la discussion à suivre, je n'en ferai pas cas dans cette étude.

${ }^{1}$ En wemegbe, les préfixes vocaliques sont $o$ et $a$.

${ }^{2}$ Dans la formule $\mathrm{C}(\mathrm{c}) \mathrm{V}$, seule $\mathrm{C}$ est concernée par les règles tonales discutées dans cet article, la consonne $\mathrm{c}(w, l, y)$ ne favorise ni ne bloque l'application des règles tonales. 
2.2 Autres structures syllabiques. Le wemegbe présente, en outre, les structures syllabiques suivantes en (4). En dehors de la structure $\mathrm{CcV}$, aucun verbal n'a été identifié au niveau des autres structures syllabiques:

$\begin{array}{lllll}\mathrm{V}: & \text { é } & \text { 'il' } & & \\ \mathrm{CV}: & \text { kú } & \text { 'meurs!' } & \text { m̌̃ } & \text { 'nous' } \\ \text { CVCV: } & \text { sónù } & \text { 'pintade' } & \text { hwēvi } & \text { 'poisson' } \\ \text { VCVCV: } & \text { àgbàzà } & \text { 'corps' } & \text { àdògò } & \text { 'ventre' }\end{array}$

\section{Point de méthodologie}

Suivant la distinction classique faite entre synchronie et diachronie préconisée par de Saussure (1916/1995), on considère un processus phonologique comme synchronique lorsqu'il est (encore) productif à un moment donné de l'axe temporel; il est diachronique, lorsqu'il fait partie (déjà) de 'l'histoire' de cette langue. Une description sera dite synchronique quand elle prend en compte les données spécifiques d'une langue dans une localité et dans un temps déterminés; i.e. un état de langue. Une étude est dite diachronique, si elle s'occupe de l'évolution de la langue à travers le temps. F. de Saussure nous met en garde contre la confusion des deux niveaux d'analyse, mais il précise qu'une bonne description diachronique suppose une série d'analyses synchroniques conséquentes. Dans ce travail, je suis conscient de cette distinction fondamentale et je ne mélangerai pas les deux niveaux d'analyse. Les tableaux de comparaison que j'utilise n'ont donc pas pour but immédiat d'identifier le proto-système dont seraient issus les systèmes particuliers (dialectaux). Ces tableaux, qui présentent des données synchroniques de differents dialectes, visent à montrer les variations interdialectales du gbe. De ce point de vue, ils peuvent se révéler très utiles dans l'analyse des phénomènes d'un dialecte particulier, lorsque le chercheur se trouve confronté par exemple à differentes solutions alternatives qui se révèlent toutes adéquates d'un point de vue descriptif. Des exemples de ce genre sont nombreux dans l'analyse des langues gbe (voir Capo 1978, 1985a, b; Gbéto 1997a, b). Dans l'identification de la forme de base du pronom clitique de la troisième personne du singulier, il a été montré que toutes les trois variantes $[i, e, \varepsilon]$ pouvaient être choisies comme formes de base en agbome, un autre dialecte gbe. La comparaison des données de l'agbome avec d'autres parlers gbe (notamment Aja et Ewe) a permis de montrer que la forme de base de ce pronom devait être /i/ dans tous les dialectes gbe. ${ }^{3}$

${ }^{3}$ En proto-gbe, ce pronom a été aussi reconstruit /i/ (voir Capo 1991). 
L'utilisation judicieuse de la comparaison dialectale permet d'identifier et de postuler des règles synchroniques. De pareils phénomènes ne sont pas du tout isolés en gbe (voir, entre autres, le phénomène d'arrondissement vocalique du gbe décrit par Capo 1986). ${ }^{4}$ Il n'est donc pas exclu qu'il en soit ainsi des phénomènes tonals. Cette méthode comparative à but synchronique a été non seulement utilisée dans la description des langues gbe, mais aussi dans celle des langues akan (voir Schachter \& Fromkin 1968), et enfin celle des langues naturelles tout court (voir Hagège \& Haudricourt 1978). Elle a été fortement recommandée par Clements (1991) qui écrit:

One of the main fruits of the pandialectal approach for phonological theory is the opportunity it provides to examine a population of closely languages and observe their patterns of variation across the same and subtly different phonological conditions. A rule that may seem uniform when only one language of the group is considered may turn out to be analyzable into a central, unmarked "core" and an extension or generalization to a new, more marked context when another language (or group of languages) is considered. The fact that some patterns of generalization are observed in preference to others suggests that rule evolution is governed by abstract structural principles that only a detailed analysis of closely related languages can bring to light. In this respect, the pandialectal approach, when based on rich materials [...], opens up the possibility of developing a typology of phonological processes that can serve as a basis for improving our models of rule markedness and historical change. [p. 16]

\section{Les Tons dans le Système Verbal}

Le wemegbe présente les tons suivants: $\mathrm{B}, \mathrm{H}, \mathrm{M}, \mathrm{HB}$ et $\mathrm{BH}$. Parmi ces tons, trois seulement sont relevés au niveau des verbaux, à savoir B, BH et $\mathrm{H}$. Je présenterai ci-dessous la distribution respective de ces deux tons. Les verbaux sont donnés dans leur forme impérative ${ }^{5}$ qui est la forme de citation du verbe en gbe.

${ }^{4}$ Toute grammaire, dont le but est de décrire la compétence du sujet parlant, doit prendre en compte aussi bien les variations intradialectales que les variations interdialectales.

${ }^{5}$ L'ordre des termes dans les langues gbe (et aussi en wemegbe) est S P O. Le prédicat P comporte le verbal et les morphèmes TAM qui sont préposés au verbal, sauf pour l'expression du morphème habituel qui est postposé au verbal dans les langues ewe, gen, mais aussi en tofin (voir Gbéto 1997a). Le morphème du passé est de la forme $\varnothing$ en wemєgbe. Il y a une différence tonale entre la forme impérative exprimée à travers le ton préfixal flottant $\mathrm{B}^{\prime}$ et la forme infinitive qui est privée de ce ton. 
4.1 La distribution du ton B. Le ton B apparait, au niveau des verbaux, sur la voyelle radicale des verbaux, quelle que soit la nature de la consonne:

$$
\begin{array}{llll}
\text { dù 'mange!' } & \text { nù 'bois!' } & \text { gbò 'coupe!' } \\
\text { xò 'bats!' } & \text { hù 'tue!' } & \text { sà 'vends!' }
\end{array}
$$

4.2 La distribution du ton $\mathrm{H}$. Le ton $\mathrm{H}$ apparât, sur la voyelle des radicaux verbaux $\mathrm{CcV}$, lorsque la consonne initiale est une [+obstruant, -voix]. Il n'est jamais relevé dans la forme impérative des radicaux verbaux dont la consonne est voisée. Les exemples en (6) et (7) sont illustratifs de cette distribution:
(6) só 'pile!'
kú 'meurs!'
kló 'lave!'
tó 'perce!'
fý́ 'fais bouillir!'
*wá
(7) *gbé
*dú

Dans ce cas, il correspond aux tons $\mathrm{H}$ et $\mathrm{BH}$ d'autres dialectes gbe. Le tableau suivant est illustratif de ce fait:

\section{Tableau 2: Correspondance $\mathrm{H}=\mathrm{BH}=\mathrm{M}$ au niveau des radicaux ver- baux CV dont $C$ est [+obstruant, -voix]}

\begin{tabular}{|c|c|c|c|c|c|}
\hline $\begin{array}{l}\text { Weme- } \\
\text { gbe }\end{array}$ & Dogbo & $\begin{array}{l}\text { Hwe- } \\
\text { gbe }\end{array}$ & $\begin{array}{l}\text { Agbome } \\
\text { (fon) }\end{array}$ & $\begin{array}{l}\text { Gun- } \\
\text { gbe }\end{array}$ & Gloses \\
\hline só & tǒ & Tō & só & só & Pile! \\
\hline kú & kǔ & $\mathrm{K} \overline{\mathrm{u}}$ & kú & kú & Meurs! \\
\hline to & tว̌ & $\mathrm{t} \bar{\sim}$ & tó & tó & Perce!(oreille) \\
\hline kpó & kpว̌ & kpj̄ & kp2́ & kp2́ & Regarde! \\
\hline
\end{tabular}

4.3 La distribution du ton BH. Il apparât au niveau des radicaux verbaux de structure $\mathrm{CV}$ dont $\mathrm{C}$ est une [+voix]:

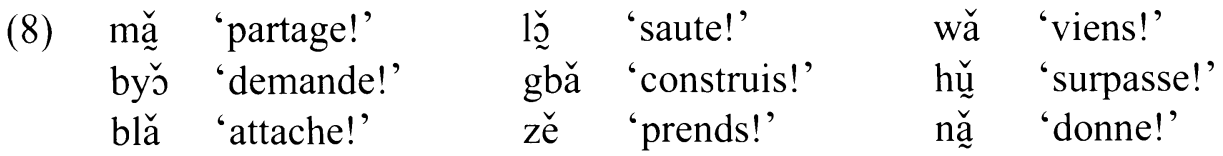


Alors que le ton $\mathrm{B}$ des radicaux verbaux correspond toujours au ton $\mathrm{B}$ dans les autres dialectes gbe, le ton $\mathrm{BH}$ correspond aux tons $\mathrm{H}, \mathrm{BH}$ et $\mathrm{M}$ des autres dialectes gbe, comme nous le montrent les tableaux suivants:

Tableau 3: Correspondance $\mathrm{BH}=\mathrm{M}$ au niveau des radicaux verbaux $\mathrm{CcV}$, dont $\mathrm{C}=[+$ obstr., +voix $]$

\begin{tabular}{|c|c|c|c|c|c|}
\hline $\begin{array}{l}\text { Weme- } \\
\text { gbe }\end{array}$ & $\begin{array}{l}\text { Xwla- } \\
\text { seme }\end{array}$ & $\begin{array}{l}\text { Kota- } \\
\text { fon }\end{array}$ & Xwla & Xwela & Gloses \\
\hline gbǎ & gbǎ & gbă & gbä & gbă & Construis! \\
\hline ǰ̃ & ǰr & jî & $\mathrm{j} \overline{1}$ & jı̌ & Gonfle! \\
\hline V̌r & V̌́ & V̌́ & $\overline{\mathrm{V}}$ & V̌1 & Pleure! \\
\hline d ̌̌ & d气 & dう̌ & $\mathrm{d} \overline{\mathrm{j}}$ & dう̌ & Dors! \\
\hline
\end{tabular}

Tableau 4: Correspondance $\mathrm{BH}=\mathrm{M}=\mathrm{H}$ au niveau des radicaux verbaux $\mathrm{CcV}$, dont $\mathrm{C}$ est [+sonant] ou [b, d]

\begin{tabular}{|c|c|c|c|c|c|}
\hline $\begin{array}{l}\text { Weme- } \\
\text { Gbe }\end{array}$ & Movolว & Dogbo & $\begin{array}{l}\text { Xwla- } \\
\text { seme }\end{array}$ & Agbome & Gloses \\
\hline wă & wá & vā & wá & wă & Viens! \\
\hline nă & ná & nāa & ná & nă & Donne! \\
\hline dŭ & dú & $\mathrm{d} \overline{\mathrm{u}}$ & dú & dǔ & Danse! \\
\hline blǎ & blá & blā & blá & blă & Lie! \\
\hline mă & má & māa & mã & mă & Partage \\
\hline
\end{tabular}

4.4 Tableau récapitulatif. La distribution des tons $\mathrm{B}, \mathrm{H}$ et $\mathrm{BH}$ au niveau des radicaux verbaux est résumée dans le tableau qui suit:

Tableau 5: Distribution des tons au niveau des verbaux de type $\mathrm{CcV}$

Radicaux verbaux commençant par T

Radicaux verbaux commençant par D

Radicaux verbaux commençant par $\mathrm{L}$

Radicaux verbaux commençant par $\mathrm{N}$

Radicaux verbaux commençant par B
$\mathrm{H} \quad \mathrm{B} \quad \mathrm{M} \quad \mathrm{BH}$

$\begin{array}{llll}+ & + & - & - \\ - & + & - & + \\ - & + & - & + \\ - & + & - & + \\ - & + & - & +\end{array}$




\section{Les Tons au Niveau du Système Nominal}

\subsection{La distribution des tons}

5.1.1 Le ton $\mathbf{H}$. Il apparaît sur la voyelle radicale nominale $\mathrm{CcV}$ des nominaux de structure $(\mathrm{V}) \mathrm{CcV}$. Dans ce cas, la consonne du radical ne peut être qu'une [+obstruant, -voix]:

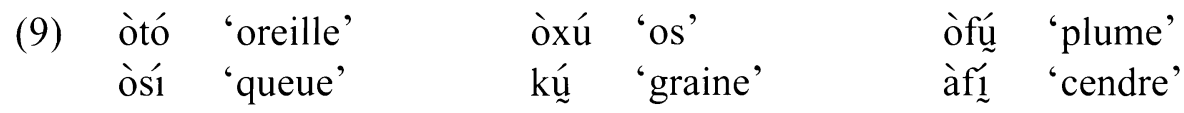

Dans ce cas, le ton $\mathrm{H}$ correspond aux tons $\mathrm{H}, \mathrm{M}$ et $\mathrm{BH}$ d'autres dialectes gbe, comme le montre le tableau suivant:

\section{Tableau 6: Correspondance $H=B H=M$ au niveau des radicaux nominaux CcV dont C est [+obstruant, -voix]}

\begin{tabular}{|c|c|c|c|c|c|}
\hline $\begin{array}{l}\text { Weme- } \\
\text { gbe }\end{array}$ & Dogbo & $\begin{array}{l}\text { Hwe- } \\
\text { gbe }\end{array}$ & $\begin{array}{l}\text { Agbome } \\
\text { (fon) }\end{array}$ & $\begin{array}{l}\text { Gun- } \\
\text { gbe }\end{array}$ & Gloses \\
\hline òtó & ètǒ & ètō & tó & òtó & oreille \\
\hline àtín & àč̀ & $\overline{\mathrm{a}} \overline{\mathrm{c}}$ & àt & àt $t_{1}$ & arbre \\
\hline òsó & ètǒ & & só & òsó & montagne \\
\hline àsú & àsǔ & āsū & àsú & àsú & mari \\
\hline
\end{tabular}

En dehors de la structure $\mathrm{VC}(\mathrm{c}) \mathrm{V}$, le ton $\mathrm{H}$ est relevé sur la dernière syllabe - $\mathrm{CV}$ des radicaux nominaux de structure VC(c)VCV. Ici, la nature de la consonne de la syllabe n'est pas déterminante pour l'occurrence de ce ton:
(10)

$\begin{array}{llll}\text { àlăká } & \text { 'joue' } & \text { àwōtí } & \text { 'nez' } \\ \text { hwèsivvó } & \text { 'soleil' } & \text { àgāsá } & \text { 'crabe' }\end{array}$
àjālākú
'éléphant'

On relève le ton $\mathrm{H}$ sur la voyelle du pronom personnel sujet troisième personne du singulier de structure $\mathrm{V}$. On relève enfin le ton $\mathrm{H}$ sur la voyelle radicale de quelques numéraux. Dans ce cas, la consonne du radical appartient à la classe des [+obstruant, -voix]: ${ }^{6}$

${ }^{6}$ Je n'ai pas pu identifier de consonnes radicales sonores dans le contexte d'un ton $\mathrm{H}$ au niveau des numéraux. Je ne sais à quoi attribuer cette lacune au niveau des numéraux de structure CVCV, surtout au niveau de la seconde consonne. 

(11) é
'il'
dōkpó
'un'
àt’ó
'cinq'

5.1.2 Le ton B. Le ton B apparaît sur la voyelle radicale $C(c) V$ des nominaux de structure $\mathrm{VC}(\mathrm{c}) \mathrm{V}$, lorsque $\mathrm{C}$ appartient à la classe des consonnes $\mathrm{B}, \mathrm{L}, \mathrm{N}$ et D. Aucune voyelle de radical nominal dont la consonne initiale est une [+obstruant, -voix] n'est intonée B:
(12) àd’̀ 'intestins'
àwà 'aile'
àlà 'chair'
ò̀ù 'sang'
àgbò
'buffle'
àd’ 'racine'
àyì
'cour'
ònù 'bouche'
àlò
'main'
(13) *àfò
*Sì
*òxwè

Au niveau des numéraux, le ton B apparât aussi au niveau des radicaux $\mathrm{CV}$, quelle que soit la nature voisée ou non de C:
(14) àwè 'deux'
àtò 'trois'
$\grave{\varepsilon} n \grave{\varepsilon}$
'quatre'

Le ton $\mathrm{B}$ apparaît enfin au niveau des pronoms personnels de type $\mathrm{V}$ et $\mathrm{CV}$ :
(15) ì
'je'
à 'tu'
$\mathrm{mì}$
'vous'

5.1.3 Le ton BH. Le ton BH apparaît au niveau des radicaux nominaux de structure $\mathrm{CcV}$, dont $\mathrm{C}$ est [+voix] et précédé ou non d'un ton $\mathrm{B}$ :
(16) àdǔ 'dent'
$\begin{array}{ll}\text { òjǒ } & \text { 'graisse' } \\ \text { àhǒ } & \text { 'nombril' } \\ \text { zã } & \text { 'nuit' }\end{array}$
lǒ
dě
ànõ
'crocodile'
ž̀ 'singe'
'un'
'sein'

Dans les cas examinés sous (16), le ton BH correspond soit au ton $\mathrm{H}$, soit au ton $\mathrm{M}$ d'autres dialectes gbe: 


\section{Tableau 7: Correspondance $\mathrm{BH}=\mathbf{H}=\mathrm{M}$ au niveau des radicaux nominaux et verbaux $\mathrm{CV}$ dont $\mathrm{C}$ est [+voix]}

\begin{tabular}{|c|c|c|c|c|c|}
\hline $\begin{array}{l}\text { Weme- } \\
\text { gbe }\end{array}$ & Dogbo & Movols & $\begin{array}{l}\text { Agbome } \\
\text { (fon) }\end{array}$ & $\begin{array}{l}\text { Gun- } \\
\text { gbe }\end{array}$ & Gloses \\
\hline àdǔ & $\bar{a} \mathrm{du}$ & ādú & àdǔ & àdù & dent \\
\hline Zăa & èzā & òză & ză & òză & nuit \\
\hline àh ِّ & èh๊̃ & àhựké & hટ̃ & àh ָِ & nombril \\
\hline ànટِ & ān & ว̄nอ̃ & nこِ & àn ِّ & sein \\
\hline lǒ & ēlō & ōló & lǒ & lǒ & crocodile \\
\hline
\end{tabular}

5.1.4 Le ton M. Le ton $M$ apparaît: avant ton $H$, sur la première voyelle radicale nominale des items polysyllabiques de type CVCV. La séquence BH est exclue au sein du radical nominal polysyllabique: ${ }^{7}$
(17)
dōkpó
ògbētó
'un'
$\begin{array}{ll}\text { kTigó } & \text { 'genou' } \\ \text { àdiś1 } & \text { 'droit' }\end{array}$
àwōtı́ 'nez'
(18) *dòkpó
*ògbètó
*kligó
*àdiś́
*àwõ ț́

5.1.5 Le ton HB. Le ton HB apparât exclusivement au niveau des radicaux non verbaux dont $\mathrm{C}$ est une [+obstruant, -voix]. Je n'ai pas identifié de radicaux non verbaux $\mathrm{CcV}$ dont $\mathrm{C}$ est non voisée et qui soit porteuse de ton $\mathrm{B}:{ }^{8}$
(19) $\begin{aligned} & \text { kô } \\ & \text { tâ } \\ & \text { to }\end{aligned}$
'vingt'
'tête'
'fleuve'
$\begin{array}{ll}\text { òkồ } & \text { 'cou' } \\ \text { kpồ } & \text { 'panthère' } \\ \text { òxwê } & \text { 'année' }\end{array}$
$\hat{a} f \hat{\jmath}$
$x \hat{\varepsilon}$
$x w l \hat{a k} o ́$
'pied'
'oiseau'
to 'fleuve'
'sel'

5.2 Tableau récapitulatif. Dans la mesure où en wemegbe, les régularités dans la distribution des tons ne s'observent qu'au niveau des morphèmes non verbaux, ${ }^{9}$

\footnotetext{
${ }^{7}$ Ces exemples contrastent avec les suivants où on remarque la présence de la séquence $\mathrm{BHH}$ : àsigé 'queue', àfífi 'cendre', àsyóví 'hache'.

${ }^{8} \mathrm{~J}$ 'ai relevé un cas de numéral après lequel on note le ton B: àt ̃̀̀.

${ }^{9}$ Au niveau des pronoms sujets au nombre de six, on relève seulement un exemple de structure $\mathrm{CV}$ portant le ton B: mì 'vous'. Malgré cela, on observe une régularité par rapport à la distribution du ton $\mathrm{H}$, lorsqu' on prend en compte les autres catégories grammaticales.
} 
je ne vais donner, dans ce qui suit, que le tableau récapitulatif qui prend en compte cette catégorie de mots.

Tableau 8: Distribution des tons au niveau des radicaux nominaux $\mathrm{CcV}$

Radicaux nominaux commençant par T

Radicaux nominaux commençant par D

Radicaux nominaux commençant par $\mathrm{L}$

Radicaux nominaux commençant par $\mathrm{N}$

Radicaux nominaux commençant par B

Radicaux nominaux commençant par/t/

$\begin{array}{lllll}\mathrm{H} & \mathrm{M} & \mathrm{B} & \mathrm{BH} & \mathrm{HB} \\ + & ?^{10} & - & - & + \\ - & ? & + & + & - \\ - & ? & + & + & - \\ - & ? & + & + & - \\ - & ? & + & + & - \\ - & ? & + & - & +\end{array}$

\section{Analyse}

6.1 Hypothèse de deux tons de base: $H$ et $B$. Dans la mesure où le ton $M$ n'est pas relevé au niveau des verbaux et où son occurrence est limitée au niveau des nominaux, il ne peut en aucun cas être choisi comme tonème. Seuls donc peuvent être phonèmes que les tons $\mathrm{B}, \mathrm{H}, \mathrm{BH}$ et $\mathrm{HB}$.

L'examen du tableau récapitulatif 5 montre qu'au niveau des radicaux verbaux, il y a contraste entre ton $\mathrm{H}$ et ton non $\mathrm{H}$, lorsque la consonne appartient à la classe T. Ce contraste disparaît devant les consonnes des classes D, L, N et B, i.e. avec les consonnes [+voix]. Dans ce cas, on observe un phénomène de distribution complémentaire entre ton $\mathrm{H}$ et ton $\mathrm{B}$. Le même phénomène de distribution complémentaire s'observe avec les tons $\mathrm{H}$ et $\mathrm{BH}$, le ton $\mathrm{BH}$ ne pouvant jamais apparaitre avec les consonnes [-voix] et le ton $\mathrm{H}$ qu'avec les radicaux verbaux et nominaux dont la consonne est non voisée. Au niveau des radicaux nominaux, on observe aussi un phénomène de distribution complémentaire entre ton $\mathrm{B}$ et ton $\mathrm{HB}$; le ton $\mathrm{B}$ ne se rencontrant jamais dans le contexte d'une consonne non voisée. Comme nous avons déjà établi le statut phonologique du ton $\mathrm{B}$, je considère le ton $\mathrm{HB}$ comme un allotone du ton B.

Dans la mesure où on observe le contraste entre ton $\mathrm{H}$ et ton non $\mathrm{H}$, i.e. B, on est en droit d'en conclure que le wemegbe comporte deux tons phonologique $/ \mathrm{H} /$ et $/ \mathrm{B} /$ et que $\mathrm{BH}$ est la réalisation du ton $/ \mathrm{H} /$ devant consonne voisée. Par ailleurs, certains pronoms de structure $\mathrm{V}$, à savoir é 'il', où on ne peut soupçonner

${ }^{10}$ Le ton $\mathrm{M}$, on l'a vu (5.1.4), n'apparât qu'au niveau des radicaux polysyllabiques, jamais au niveau des monosyllabiques. C'est ce qui justifie les interrogations dans la colonne du ton $\mathrm{M}$. 
l'action quelconque d'une consonne à moins que ce soit celle de la consonne qui suit (ce qui est invraisemblable, si l'on en crôit Hombert (1978), portent un ton H. Si l'on choisit $\mathrm{BH}$ comme représentant le tonème $/ \mathrm{H} /$, on serait obligé d'écrire une règle ad hoc d'effacement du ton B. Ce qui ne serait pas le cas, si l'on choisit l'allotone $\mathrm{H}$.

Mais que dire du ton M? Nous avons vu que ce ton n'apparât qu'avant ton $\mathrm{H}$ et que la séquence tonale $\mathrm{BH}$ n'est pas permise au niveau des radicaux polysyllabiques. Je voudrais donc considérer le ton $\mathrm{M}$ comme une réalisation du ton $\mathrm{B}$ dans le contexte d'un ton $\mathrm{H}$. On est donc en droit de dire que le wemsgbe oppose un ton $\mathrm{H}$ à un ton non $\mathrm{H}$, symbolisé respectivement par $\mathrm{H}$ et $\mathrm{B}$. Les autres tons, à savoir HB, M et BH, seront considérés comme des allotones de ces deux tons de base et il s'agit de dégager les règles tonales de leur réalisation.

\subsection{Réalisations des tonèmes $\mathrm{H}$ et $\mathrm{B}$}

\subsubsection{Le ton $B H$ en tant que réalisation du ton $H$ et l'hypothèse d'un préfixe} tonal $\mathbf{B}^{\prime}$. On a vu que le ton $\mathrm{H}$ des radicaux verbaux et nominaux est réalisé $\mathrm{BH}$ devant consonne voisée. J'avais alors émis l'hypothèse que ces radicaux portaient en structure sous-jacente un tonème $H$. Sur cette base, on peut poser une règle d'abaissement $\mathrm{du}$ ton radical $\mathrm{H}$ et la question est de savoir les motivations phonétiques de cette règle. Rappelons qu'au niveau des nominaux, le préfixe vocalique est toujours intoné B et que, au niveau des verbaux, nous nous trouvons en face d'une forme impérative. On est alors tenté de poser pour toute forme (verbale ou nominale) un ton préfixal B'. C'est cette position qu'ont adoptée Stahlke (1971a,b, 1976) pour le kpando et le yoruba, Capo (1977) pour le gengbe et le wacigbe. Ce point de vue a été défendu pour l'ensemble des parlers gbe par Gbéto (1995). Au niveau des verbaux, le ton préfixal B' représente le morphème de l'impératif (voir aussi Capo \& Kossouho 2003, Gbéto 2003). C'est probablement l'absence de ce ton préfixal au niveau du pronom $\mathrm{V}$ qui explique la présence du ton $\mathrm{H}$ au niveau de /é/ [é] 'il'. On peut alors écrire la règle d'abaissement comme une règle qui propage le ton préfixal $\mathrm{B}^{\prime}$ sur le ton $\mathrm{H}$ radical:

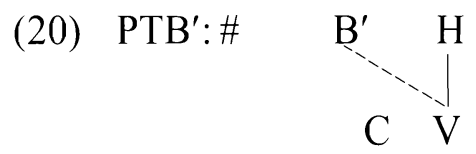

Le préfixe tonal ne peut se propager qu'à travers les $[\mathrm{C},+$ voix $]$. Les raisons qui motivent la non-propagation du ton préfixal $\mathrm{B}^{\prime}$ à travers les consonnes [+obstruant, -voix] seront discutées au 6.2.2. 


\begin{tabular}{|c|c|c|c|c|}
\hline (21) a. / 'wá/ & & $\rightarrow$ & [wǎ] & 'viens!' \\
\hline b. /àví/ & & $\rightarrow$ & [àvı̌i] & 'pleurs' (nominal) \\
\hline c. / zé/ & & $\rightarrow$ & [ž̌ ] & 'prends' \\
\hline d. /àdú/ & & $\rightarrow$ & [àdǔu & 'dent' \\
\hline e. /òtó/ & $\rightarrow$ & *òtǒ $\rightarrow$ & [òtó] & 'oreille' \\
\hline f. /òfú// & $\rightarrow$ & *òfũ $\rightarrow$ & [òfúu & 'plume' \\
\hline
\end{tabular}

\subsubsection{Réalisations du ton B}

6.2.2.1 Le ton HB et l'hypothèse d'insertion du ton $\mathbf{H}$. Nous avons vu que le ton $\mathrm{HB}$ est conditionné phonétiquement. Il n'est relevé qu'avec des radicaux de type $\mathrm{CcV}$ dont $\mathrm{C}$ est une obstruante non voisée. C'est là un type particulier de relation entre tons et consonnes reconnue déjà par plusieurs chercheurs ayant travaillé sur les langues de l'Afrique de l'ouest (voir, entre autres, Ansre 1961; Stahlke 1971a,b; Hombert 1978; Capo 1977; Bole-Richard 1983; Hyman \& Schuh 1974; Hyman \& Mathangwane 1998, Gbéto 1995, 1997a, 1998/99, 2002a, b; Bradshaw 1999; Odden 2004). Les obstruantes voisées ont pour effet de rabaisser $^{\prime \prime}$ la réalisation tonale suivante, alors que les obstruantes non voisées ont pour effet de la rehausser. Pour capturer cette relation, je voudrais faire l'hypothèse qu'une consonne rehaussante, symbolisée ici par R, crée un ton $\mathrm{H}$ dans sa structure squelettale, comme le montre la règle ITH (Insertion du ton $\mathrm{H}$ ):
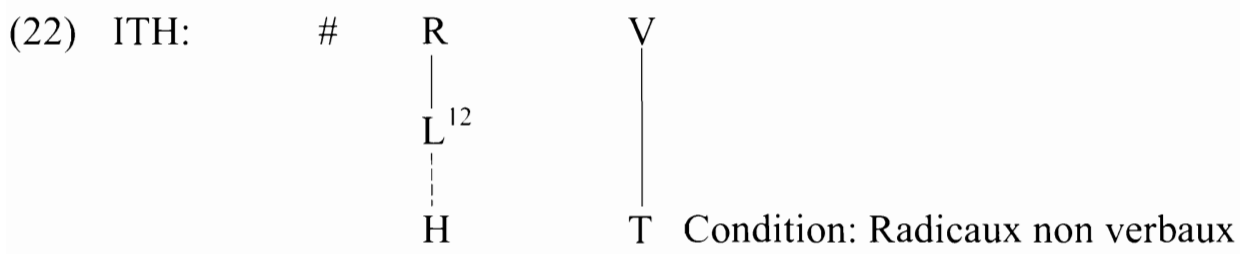

Cette règle stipule que les consonnes rehaussantes (non voisées) insèrent un ton $\mathrm{H}$ devant toute voyelle portant un des tonèmes quelconques (i.e. $\mathrm{B}$ ou $\mathrm{H}$ ) de la langue.

"Dans la terminologie anglophone, ces consonnes sont appelées "depressor consonants". Faute de mieux, je traduirai ce terme par "consonnes abaissantes" dans les lignes qui suivent. Pour les obstruantes non voisées qui rehaussent les tons, on les appellera "consonnes rehaussantes".

${ }^{12} \mathrm{~L}$ désigne ici le nœud Laryngal. 
Lorsque le ton vocalique est $\mathrm{B}$, le ton $\mathrm{H}$ inséré se propagera à sa droite sur ce ton $\mathrm{B}$ selon la règle PTIH (Propagation du Ton Inséré $\mathrm{H}$ ). Cela signifie que le ton vocalique dans les formes [cv] portent en réalité un ton /B/ dans leur forme de base. PTIH peut être formulé comme suit:

(23)

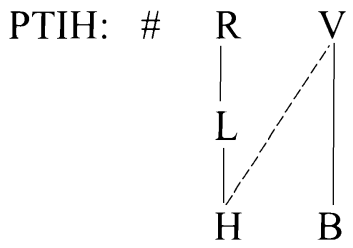

Ainsi, [àfô $]^{13}$ peut être dérivé comme suit:

(24)

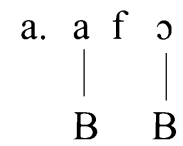

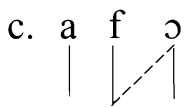

B H B

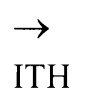

[àfô] $\rightarrow$

PTIH

B H B

Cette analyse est supportée par la comparaison dialectale, puisque, dans les mêmes conditions énumérées ci-dessus, les autres dialectes gbe présenteront les ton B ou M (voir le tableau 9 ci-dessous).

\section{Tableau 9: Correspondance $\mathrm{HB}=\mathrm{B}=\mathrm{M}$ au niveau des radicaux non verbaux $\mathrm{CcV}$ dont $\mathrm{C}$ est [+obstruant, -voix]}

\begin{tabular}{|c|c|c|c|c|}
\hline $\begin{array}{l}\text { Wems- } \\
\text { gbe }\end{array}$ & $\begin{array}{l}\text { Agbome } \\
\text { (fon) }\end{array}$ & Dogbo & Movols & $\begin{array}{l}\text { Gun- } \\
\text { gbe }\end{array}$ \\
\hline àfô & àfò & $\bar{a} f \bar{s}$ & ว̀f’ & \\
\hline$\hat{n}$ & kpj̀ & ēkpj̄ & ōkp̄̄ve & òkpò \\
\hline 10 & xwè & èxwē & òxwè & òxwè \\
\hline
\end{tabular}

13 J'ai montré, ailleurs (voir Gbéto 1997a), que le préfixe vocalique nominal est extratonal au sens de Pulleyblank (1986) en gbe. De ce point de vue, la consonne radicale est supposée être en position initiale. 
L'hypothèse selon laquelle les [+obstruant, -voix] insèrent un ton $\mathrm{H}$ est supportée non seulement par la comparaison dialectale où le ton HB des radicaux nominaux correspond au ton $\mathrm{B}$ d'autres dialectes gbe, mais aussi par les phénomènes de redoublement verbal et de composition nominale:
a. /xò/ 'battre' $\rightarrow$ xìxò $\rightarrow$ 'xixò $\rightarrow$ xíxò $\rightarrow$ [xíxō] 'le fait de battre' /sà/ 'vendre' $\rightarrow$ sìà $\rightarrow$ ' sìsa $\rightarrow$ śisà $\rightarrow$ [sísā] 'le fait de vendre'
b. /xwlà + kó/ $\rightarrow$ 'xwlà kó
$\rightarrow$ [xwlâkó $]$
'sel' 14
Pla sable

La règle ITH au niveau des radicaux $\mathrm{CcV}$ explique pourquoi, d'un point de vue formel, on n'observe pas de ton modulé $\mathrm{BH}$, résultat de la propagation du ton préfixal $\mathrm{B}^{\prime}$ sur le ton $\mathrm{H}$ suivant, après $[\mathrm{C}$, -voix]. Cette propagation croiserait la ligne d'association qui relie $\mathrm{C}$ au ton $\mathrm{H}$; ce qui est naturellement interdit par la convention de l'association (Goldsmith 1976/1979, 1990). Ainsi, /òtó/ 'oreille' ne se réalisera pas *[òtǒ] mais [òtó]:

(26) Dérivation de /òtó/ 'oreille'
a. ${ }_{\mathrm{B}}^{\mathrm{O}}$

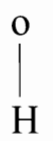
$\overrightarrow{\text { ITH }}$
b.

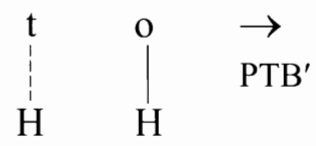

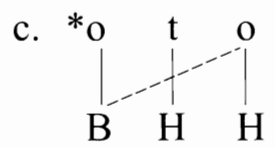

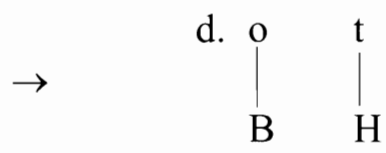
$\left.\left.\right|_{\mathrm{H}} ^{\mathrm{t}} \quad\right|_{\mathrm{H}} ^{\mathrm{O}}$
[òtó] 'oreille'

6.2.2.2 Le ton $M$, réalisation du ton $B$ devant ton $H$. Il faut postuler une règle de relèvement du ton $\mathrm{B}$ avant ton $\mathrm{H}$ au niveau des radicaux non verbaux pour expliquer le ton $\mathrm{M}$ observé avant ton $\mathrm{H}$ au niveau d'item polysyllabiques:

(27)

$\mathrm{RTB}_{2}: \quad \mathrm{B} \rightarrow \mathrm{M} /$ $\mathrm{H}$

\footnotetext{
${ }^{14}$ L'ethnie Pla mâtrise la technique d'extraction du sel dans l'ancien royaume du Danhomê. Le sel étant un mot tabou, on le désigne par l'euphémisme 'sable des Pla'.
} 

a. /ḋ̀ggbé/ $\rightarrow$
nغ̄gbé $\rightarrow$
[nēgbé]
'dos'
b. /dụikú / $\rightarrow$
nù̀kú $\rightarrow$
[nūkưu]
'oeil'
c. làwờti/l $\rightarrow$
[àwờút
'nez'

6.3 Les tons dans le redoublement verbal. Le redoublement verbal est un processus morphologique très productif en gbe (voir Capo 1989). Il consiste à préfixer à la base verbale $[\mathrm{C}(\mathrm{c}) \mathrm{V}]$, une base sous-spécifiée [Ci], où [C] est la copie de la consonne initiale. La voyelle /i/ assimile les traits de nasalité, de labialité de la voyelle radicale. Ce qui nous préoccupe ici est le phénomène tonal induit par le redoublement. La question que je me pose est la suivante: le ton de la voyelle du réduplicatif est-il le ton de la voyelle radicale? En d'autres termes, y at-il une règle qui propage le ton radical sur la voyelle non spécifiée sur le plan tonal? Avant de répondre à cette question, voyons dans la réalité comment le processus a lieu en wemegbe. Je voudrais rappeler que le verbe comporte deux morphèmes: une partie radicale $[\mathrm{C}(\mathrm{c}) \mathrm{V}]$ (qui est la forme infinitive du verbe) et le ton préfixal B'qui est le ton de l'impératif.

Lorsque la voyelle radicale est intonée $\mathrm{H}$ et que la consonne de la syllabe est une [+obstruant, -voix], le réduplicatif portera un ton $\mathrm{H}$ :

(28)
a. \fyé/ $\rightarrow$
[fifyé]
'le fait de faire bouillir'
b. Гsó/ $\rightarrow$
[sísó]
c. / kú/ $\rightarrow$
[kúkú]
'le fait de piler'
d. / tó/ $\rightarrow$

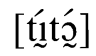
'le fait de mourir'
'le fait de percer'

Lorsque la voyelle radicale est intonée $H$ et que la consonne de la syllabe est une [(+obstruant), +voix], le réduplicatif portera un ton $\mathrm{B}$ pendant que le radical verbal portera un ton $H$ :
a /`yó/ $\rightarrow$
[ỳyó]
'le fait de fondre'
b. / zé/ $\rightarrow$
[zizé]
'le fait de prendre'
c. /'wá/ $\rightarrow$
[wìá]
'le fait de venir'
d. Г'gbá/ $\rightarrow$
[gbigbá]
'le fait de construire'

Lorsque la voyelle radicale est intonée B et que la consonne de la syllabe est une [(+obstruant), +voix], le réduplicatif portera un ton B pendant que le radical verbal lui aussi portera un ton $\mathrm{B}$ : 
(30)
a. / dù $\rightarrow \quad$ [dùdù]
'le fait de manger'

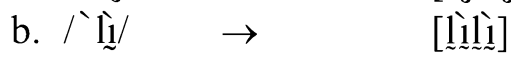
'le fait de nager'
c. $/ \mathrm{ji} / \quad \rightarrow \quad$ [jiji]
'le fait d'accoucher'
d. $/ \mathrm{zùj} / \rightarrow$
[zù̃zù]
'le fait d'insulter'

Lorsque la voyelle radicale est intonée B et que la consonne de la syllabe est une [+obstruant, -voix], la forme redoublée du radical verbal portera le schème tonal HM:
(31)

\begin{tabular}{|c|c|c|}
\hline a. / sà/ & $\rightarrow$ & [ś́sā] \\
\hline b. / kù & $\rightarrow$ & [kúk $\left.{ }^{\prime} \bar{\sim}\right]$ \\
\hline /xà/ & - & [x́́xā] \\
\hline d. / sè/ & & ['śsē] \\
\hline
\end{tabular}
'le fait de couler (fleuve)'
'le fait de creuser'
'le fait de compter'
'le fait d'entendre'

En examinant les formes en (28)-(31), on est tenté de poser les hypothèses suivantes: le ton du réduplicatif verbal porte le ton par défaut $\mathrm{B}$, identique au ton préfixal; le ton du réduplicatif est une copie du ton radical.

La première hypothèse permet de rendre compte des redoublements verbaux en (28)-(29), puisqu'elle dériverait [zizé] de /zé/ et [dùdù] de /dù/. Mais elle dériverait faussement $*[$ kùkú] de $/$ kú/ et $*[$ sìsà] de /sà/. Pour dériver la forme correcte, on pourra imaginer le scénario suivant. Puisque le verbe redoublé ne fait pas partie de la classe des verbaux, ITH puis PTIH s'appliquent. La simplification du contour tonal HB permettrait alors de dériver [kúkú] de /kùkú/, mais également [śsà] de /sisà/. Il reste cependant à se demander comment justifier le relèvement du ton $\mathrm{B}$ du radical verbal. On pourra ensuite faire propager le ton $\mathrm{H}$ de [sí-] sur le ton suivant et dériver la séquence HM de H-HB.

La question se pose de savoir s'il n'y a pas une grammaire plus simple pour rendre compte des faits observés. On fera remarquer qu'en wemєgbe, mais aussi en gbe, le réduplicatif copie les traits de nasalité (par exemple tó 'percer' sera redoublé en [țítõ] en passant par títơ) et de labialité (lorsque la voyelle est haute, par exemple kú 'mourir' sera redoublé en [kúkú] en passant par kíkú) de la voyelle radicale. Pourquoi le même phénomène ne s'observerait-il pas lorsqu'il s'agit des faits tonals? Je voudrais donc opter pour la seconde hypothèse et dire que le wemsgbe a une règle qui propage le ton radical sur la voyelle. Cette règle, je la formule comme PTR (Propagation du Ton Radical): 
(32) PTR: C

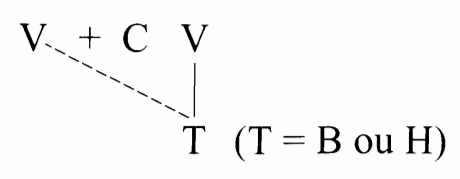

Ainsi, les radicaux verbaux de type /cv̀/, avec $\mathrm{C}=[+$ voix $]$, auront pour forme redoublée [cv̀cv̀] (par exemple dù 'mange!' sera redoublé en dùdù). Lorsque les radicaux sont de type [ć́], le redoublement est de la forme [cv́cv́] (par exemple kú 'mourir' sera redoublé en kúkú). Les radicaux verbaux de type [č̌] redoublent en [cìcv́] (par exemple gbă 'construis!' sera redoublé en gbígbá). Dans cette forme, on voit que le radical est intoné $\mathrm{H}$ et ce ton $\mathrm{H}$ représente le ton de base, celui de la forme infinitive du verbe. Le redoublement /cv́/ de cette forme donnant toujours cv́cv́, il s'agit d'expliquer pourquoi, en lieu et place, nous avons [cìcv́]. Comme la forme redoublée est un élément non verbal, je fais l'hypothèse qu'elle est affectée d'un ton préfixal B'. On pourra alors expliquer l'abaissement $\mathrm{du}$ ton $\mathrm{H}$ du réduplicatif par une règle de propagation du ton préfixal $\mathrm{B}^{\prime}$. Cette règle est une forme remaniée de $\mathrm{PTB}^{\prime}$ :

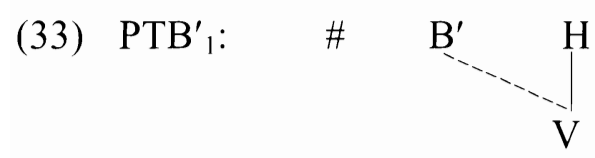

Si l'hypothèse du préfixe tonal est vraie, comment expliquer que la forme redoublée de /kú/ 'mourir' ne donne pas *[kùkú] mais plutôt [kúkú]; en d'autres termes pourquoi $\mathrm{PTB}_{1}{ }_{1}$ ne s'applique pas ici. Si on sait que les consonnes voisées insèrent un ton $\mathrm{H}$, on pourra expliquer la forme [kúkú] par l'impossibilité du ton préfixal $\mathrm{B}^{\prime}$ de se propager à sa droite pour dériver la forme incorrecte *[kùkú], cette propagation devant entrâner le croisement des lignes d'association interdit par la convention de l'association.

Il reste à expliquer comment dériver cv́c̄ de cì, i.e. [ś́sā] de /sà/ 'achète!'? Dans la mesure où la forme redoublée est un item verbal et que l'insertion du ton $\mathrm{H}$ s'observe en position initiale de radical, je fais l'hypothèse que /sà/ passera par une étape intermédiaire qui se manifestera par l'application de ITH et PTIH qui dérivera [sâ]. Par PTR, le premier versant du ton HB se propage sur la voyelle du réduplicatif: ${ }^{15}$ ce qui permet de dériver [ś́sâ]. Ensuite, la séquence H-HB (sísâ) se réalisera H-M [sísā].

${ }^{15}$ Je remercie David Odden pour m'avoir suggéré cette hypothèse que je trouve attrayante et dont j'endosse l'entière responsabilité. 
6.4 Le traitement d'exception. ITH et PTIH, on l'a vu, s'appliquent aux radicaux non verbaux pour dériver le ton $\mathrm{HB}$ du ton $/ \mathrm{B} /$. Il existe pourtant un cas de morphème non verbal, le numéral àt ָ̃ 'trois', dont la consonne, qui est une consonne rehaussante, ne module pas le ton B. L'existence d'exception au niveau de radicaux non verbaux est un indice que ITH et $\mathrm{PTIH}$ sont des règles de nature phonologique. Si cette règle était une règle phonétique, on ne s'attendrait pas à avoir d'exception, toutes les consonnes rehaussantes devant favoriser ITH et PTIH; puisque je ne m'explique pas pourquoi /kò/ [kô] 'vingt' modulera le ton B en $\mathrm{HB}$ et pas /àtว̃/ [àt’̃]. La non-application de ITH et PTIH au niveau de [àtว̃] trouve-t-elle son explication dans le fait que /t/, qui est la consonne coronale par défaut ne soit pas transparente à l'application de ces règles au niveau dues numéraux? L'unicité ${ }^{16}$ de l'exemple ne permet pas de répondre avec satisfaction à cette question.

\section{Conclusion}

Cette étude nous a permis de voir que, comme dans les autres langues gbe, le wemegbe oppose deux tons sous-jacents symbolisés par B et $\mathrm{H}$. Les autres tons sont des tons dérivés de ces deux tons de base. Ainsi, le ton $\mathrm{M}$ est une réalisation $\mathrm{du}$ ton $/ \mathrm{B} /$ en contact avec le ton $\mathrm{H}$. La modulation tonale $\mathrm{BH}$ s'explique par une règle de propagation du ton (préfixal) $\mathrm{B}^{\prime}$. Quant à la modulation tonale $\mathrm{HB}$, qui apparâit au niveau des nominaux, elle s'explique par une règle d'insertion avant ton $\mathrm{B} \mathrm{du}$ ton $\mathrm{H}$, interprétation phonétique $\mathrm{du}$ non-voisement de la consonne. L'insertion du ton consonantique $\mathrm{H}$ explique pourquoi les consonnes sourdes bloquent la propagation du ton préfixal B' à travers elles. A l'issue de la description

${ }^{16}$ Je n'ai identifié que deux numéraux comportant la consonne coronale par défaut $/ \mathrm{t} /$. Il s'agit de /àtỡ / 'cinq' et /àtว̃/ 'trois'. Ces deux numéraux ont la même correspondance tant au niveau segmental qu'au niveau prosodique dans les autres variétés dialectales gbe.

Le lecteur anonyme estime qu'on pourrait rendre compte de cet exemple en le considérant comme une exception occasionnelle. L'argument de la transparence des consonnes coronales évoqué ici ne serait donc pas justifié, puisque la transparence des consonnes coronales est du ressort de l'harmonie vocalique faisant intervenir la localisation. Même si cela est vrai, on relève, en gbe, plus particulièrement en tofingbe de Ganvie (voir Gbéto 2004), des cas de transparence (ou de non de transparence) de la coronale / $t$ / aux règles tonales. En tofingbe de Ganvié par exemple, la coronale / $\mathrm{t} /$ est transparente à la règle d'abaissement du ton radical $\mathrm{H}$ applicable uniquement au niveau des radicaux $\mathrm{CcV}$, dont $\mathrm{C}$ est [+voix]. L'hypothèse de la non-transparence au niveau de numéraux semble, à mon avis, donc justifiée. 
de la tonologie synchronique du wemegbe, nous voudrions suggérer que les unités porteuses de tons dans les langues naturelles sont non seulement les voyelles, mais aussi les consones radicales (Newmann 1996, Odden 1996).

\section{RÉFÉRENCES}

Ansre, Gilbert. 1961. The Tonal Structure of Ewe. Hartford Studies in Linguistics 1. Hartford, Conn.: Hartford Seminary Foundation.

Bole-Richard, Rémy. 1983. Systématique phonologique et grammaticale d'un parler Ewe: le Gen-Mina du Sud-Togo et Sud-Bénin. Paris: L'Harmattan.

Bradshaw, Mary. 1999. A Crosslinguistic Study of Consonant-Tone Interaction. Columbus: Ohio State University, Ph. D. dissertation.

Capo, Hounkpati B. C. 1977. Etude phonologique comparée du wacigbe et du gengbe. Cotonou: Université Nationale du Bénin, Mémoire de maitrise.

Capo, Hounkpati B. C. 1978. "A propos des corrélations de palatalisation et de labialisation en fon". Annales de l'Université d'Abidjan (série H, Linguistique) 11:5-19.

Capo, Hounkpati B. C. 1981. A Diachronic Phonology of the 'Gbe' Dialect Cluster. Legon: University of Ghana, Ph. D. Dissertation.

Capo, Hounkpati B. C. 1983. '“I' et 'U' en Hwe et leur place dans la reconstruction du proto-gbe". Journal of West African Languages XIII, 1: 19-30.

Capo, Hounkpati B. C. 1985a. "Determining the third personal singular object pronoun in Gbe". West African Languages in Education, ed. by Kay Williamson. Wien: Afro-Pub. Pp. 106-131.

Capo, Hounkpati B. C. 1985b. "Palatalisation' synchronique en Gbe”. Afrika und Übersee 68: 187-206. 
Capo, Hounkpati B. C. 1986. "Vowel roundness in Gbe: a pandialectal approach”. Journal of West African Languages 16: 15-36.

Capo, Hounkpati B. C. 1989. "L'assimilation d'arrondissement dans le redoublement des formes verbales en Gbe". Linguistique Africaine 3: 19-42.

Capo, Hounkpati B. C. 1991. A Comparative Phonology of Gbe. Berlin \& Garome: Foris \& Labo-Gbe (Int.).

Capo, Hounkpati B. C. 2000. The New Ewe Orthography. Cape Town \& Garome: Casas \& Labo-Gbe (Int.).

Capo, Hounkpati B. C. \& F. Félicité Kossouho. 2003. "De quelques spécificités phonologiques de l'Ajlagbe: un parler gbe". Linguistique Africaine 23: 19-51.

Clements, G. N. 1991. "Foreword” to Capo 1991. Pp. xv-xvi.

C. N. L. (Commission Nationale de Linguistique). 1983. Atlas Sociolinguistique du Bénin. Institut de Linguistique Appliquée / Agence de Coopération Culturelle et Technique: Abidjan/Paris.

Gbéto, Flavien. 1995. "Quelques aspects comparatifs et diachroniques de la tonologie du gbe". Afrika und Übersee 78: 73-99.

Gbéto, Flavien. 1997a. Le maxi du Centre-Bénin et du Centre-Togo. Köln: Rüdiger Köppe Verlag.

Gbéto, Flavien. 1997b. "Assimilation du pronom substitutif en Gbe et phénomènes apparentés revisités". Afrikanistische Arbeitspapiere 49: 105132. Köln: Institut für Afrikanistik, University of Köln.

Gbéto, Flavien. 1998/99. "Description et Hypothèses sur la nature de l'UPT (Unité Porteuse de Ton): l'exemple des langues gbe". Journal of West African Languages 27.2: 3-20. 
Gbéto, Flavien. 2000. Les emprunts linguistiques d'origine européenne en Fon (Nouveau Kwa, Gbe: Bénin): une étude de leur intégration au plan phonéticophonologique. Köln: Rüdiger Köppe Verlag.

Gbéto, Flavien. 2002a. "Une esquisse de la tonologie du məvəlっ, dialecte gbe du Sud-Bénin". Cahiers d'Etudes Linguistiques 6: 47-59.

Gbéto, Flavien. 2002b. "Les consonnes voisées 'implosives' et leurs rôles dans la tonogenèse et la nasalité des consonnes dans quelques langues Volta-Congo". Gbe Studies/Etudes Gbe 1:7-31.

Gbéto, Flavien. 2003. "Le système tonal du dogbo, variété dialectale Aja du SudBénin (New Kwa, Gbe) entre le passé et le présent". Linguistique Africaine 23: 75-108.

Goldsmith, John A. 1976/1979. Autosegmental Phonology. Cambridge, Mass.: MIT, PhD. dissertation. Distributed by Indiana University Linguistics Club: Bloomington.

Goldsmith, John A. 1990. Autosegmental and metrical phonology. Cambridge: Blackwell.

Goldsmith, John A. (ed.). 1996. The Handboook of Phonological Theory. Cambridge: Blackwell.

Hagège, Claude \& André Haudricourt. 1978. La phonologie panchronique. Paris: PUF.

Hombert, Jean-Marie. 1978. "Consonant types, vowel quality and tone". Tone: a Linguistic Survey, ed. by Victoria Fromkin. New York: Academic Press. Pp. 77-111.

Houndékan, Cosme Z. 1983. Contribution à l'étude phonologique du wemsgbe: le parler d'Ajowun, Mémoire de mâtrise, Université Nationale du Bénin, Abomey-Calavi.

Hyman, Larry \& Joyce T. Mathangwane. 1998. "Tonal Domains and Depressor Consonants in Ikalanga". Theoretical Aspects of Bantu Tones, ed. by Larry 
Hyman \& Charles Kisseberth. Stanford: CSLI, Stanford University. Pp. 195229

Hyman, Larry \& Russell Schuh. 1974. "Universals of tone rules: evidence from West Africa". Linguistic Inquiry 5: 81-115.

Mukarovsky, G. Hans. 1969. "Zur Struktur des Ewe". Wort und Religion, ed. by Hans-Jürgen Greschat \& Hermann Jungraithmayr. Stuttgart: Evangelischer Missionsverlang Gmbh. Pp. 107-126.

Newmann, Paul. 1996. "Hausa tonology: complexities in an 'easy' tone language". in Goldsmith 1996. Pp. 762-781.

Odden, David. 1996. "Tone: African Languages”. in Goldsmith 1996. Pp. 444475.

Odden, David. 2004. "The Verbal Tone System of Zina Kotoko". Some Aspects of the Grammar of Zina Kotoko, ed. by Bodil Kappel Schmidt, David Odden \& Anders Holmberg. Lincom Studies in African Linguistics 54. München: Lincom Europa. Pp. 15-34.

de Saussure, Ferdinand. 1916/1995. Cours de Linguistique Générale. Paris: Payot.

Schachter, Paul \& Victoria A. Fromkin. 1968. "A Phonology of Akan: Akuapem, Asante, Fante". Working Papers in Phonetics 9, UCLA.

Stahlke, Herbert. 1971a. Topics in Ewe phonology. Los Angeles: UCLA, Ph. D. Dissertation.

Stahlke, Herbert. 1971b. "The noun prefix in Ewe". Studies in African Linguistics, Supplement 2: 141-159.

Stahlke, Herbert. 1976. "The noun prefix in Yoruba". Studies in African Linguistics, Supplement 6: 243-253. 
Stewart, John M.. 1989. "Kwa”. The Niger-Congo Languages, ed. by John Bendor-Samuel. New York: University Press of America. Pp. 217-245

Stewart, John M. 1994. "Review Article: The Comparative Phonology of Gbe and its Significance for that of Kwa and Volta-Congo". Journal of African Languages and Linguistics 15: 175-193.

Westermann, Diedrich. 1927. Die westlichen Sudansprachen und ihre Beziehungen zum Bantu. Berlin: Walter de Gruyter.

Westermann, Diedrich. 1930. A Study of the Ewe language (translated by B. Smith). London: Oxford University Press.

Westermann, Diedrich. 1947. Der Wortbau des Ewe. Abh. der Preussischen Akad. der Wiss. Phil.-Klasse, Nr 9. Berlin: Verlag der Akademie der Wissenschaften.

Williamson, Kay. 1989. "Niger-Congo overview". The Niger-Congo Languages, ed. by John Bendor-Samuel. New York: University Press of America. Pp. 345 .

Dynamique des Langues et Cultures a Calavi

(Labodylcal)

Campus Universitaire à Abomey-Calavi

01 B.P. 526 Cotonou

République du BENIN

fgbeto@bj.refer.org
[Received October 12, 2003 accepted August 8, 2004] 